\title{
Detection and parameter estimation of binary neutron star merger remnants
}

\author{
Paul J. Easter®, ${ }^{1,2, *}$ Sudarshan Ghonge, ${ }^{3}$ Paul D. Lasky, ${ }^{1,2}$ Andrew R. Casey, ${ }^{1,4}$ James A. Clark, ${ }^{3}$ \\ Francisco Hernandez Vivanco $\odot,{ }^{1,2}$ and Katerina Chatziioannou ${ }^{5}$ \\ ${ }^{1}$ School of Physics and Astronomy, Monash University, VIC 3800, Australia \\ ${ }^{2}$ OzGrav: The ARC Centre of Excellence for Gravitational Wave Discovery, Clayton VIC 3800, Australia \\ ${ }^{3}$ Center for Relativistic Astrophysics and School of Physics, Georgia Institute of Technology, \\ Atlanta, Georgia 30332, USA \\ ${ }^{4} A R C$ Centre of Excellence for All Sky Astrophysics in 3 Dimensions (ASTRO 3D), \\ Canberra, ACT 2611, Australia \\ ${ }^{5}$ Center for Computational Astrophysics, Flatiron Institute, \\ 162 5th Avenue, New York, New York 10010, USA
}

(Received 8 June 2020; accepted 27 July 2020; published 14 August 2020)

\begin{abstract}
Detection and parameter estimation of binary neutron star merger remnants can shed light on the physics of hot matter at supranuclear densities. Here we develop a fast, simple model that can generate gravitational waveforms, and show it can be used for both detection and parameter estimation of postmerger remnants. The model consists of three exponentially damped sinusoids with a linear frequency-drift term. We test the model against nine equal-mass numerical-relativity simulations selected for emission of gravitational waves for $\gtrsim 25 \mathrm{~ms}$. The median fitting factors between the model waveforms and numerical-relativity simulations exceed 0.90 . We detect remnants at a postmerger signal-to-noise ratio of $\geq 7$ using a Bayes-factor detection statistic with a threshold of 3000. We can constrain the primary postmerger frequency to $\pm_{1.2}^{1.4} \%$ at postmerger signal-to-noise ratios of 15 with an increase in precision to $\pm_{0.2}^{0.3} \%$ for postmerger signal-to-noise ratios of 50. The tidal coupling constant can be constrained to $\pm_{12}^{9} \%$ at postmerger signal-to-noise ratios of 15 , and $\pm 5 \%$ at postmerger signal-to-noise ratios of 50 using a hierarchical inference model.
\end{abstract}

DOI: $10.1103 /$ PhysRevD.102.043011

\section{INTRODUCTION}

Gravitational waves have been directly detected from the inspiral of binary neutron star mergers [1,2]. The postmerger remnant may promptly collapse into a black hole, or form a hot, differentially rotating neutron star [3,4], which emits gravitational waves, e.g., [5-8]. Numerical-relativity simulations of postmerger remnants show relationships between the gravitational-wave spectra and a number of progenitor properties through quasiuniversal relationships, e.g., [9-18]. Of particular interest is the relationship between the progenitor tidal coupling constant and the primary postmerger oscillation frequency for baryonic equations of state $[14,16,17,19]$, which can be used to place constraints on the tidal coupling constant.

Gravitational-wave spectra generated from numericalrelativity simulations show consistent features related to the dynamics of the surviving remnant. A dominant peak, designated as $f_{\text {peak }}$ [20], is produced by the fundamental oscillations of the bar-mode deformed postmerger remnant, e.g., $[9,21-24]$. The frequencies of four possible peaks can

paul.easter@monash.edu be labeled as $\left(f_{2-0}, f_{\text {spiral }}, f_{\text {peak }}, f_{2+0}\right)$ in ascending order [15]. The peaks at frequencies $f_{2-0}, f_{2+0}$ may result from coupling between a quasiradial oscillation mode and $f_{\text {peak }}$ [25]. The peak at frequency $f_{\text {spiral }}$ may result from the slower rotation rate of tidally deformed matter at the outer edges of the postmerger remnant [15]. See Refs. [13,16] for an alternative proposed explanation of the frequency peaks.

In this paper, we develop a Bayesian detection and parameter-inference pipeline. Normally these pipelines require a large bank of waveforms. Numerical-relativity simulations cannot be used to generate these waveforms as each simulation requires $\sim \mathcal{O}\left(10^{5}\right)$ CPU hours to complete [16]. We develop a fast, simple model of gravitational waves for postmerger remnants that phenomenologically incorporates the main frequencies previously mentioned. Our model produces waveforms in a time frame that is suitable for use in detection and parameter estimation of binary neutron star postmerger remnants.

We match nine equal-mass numerical-relativity waveforms with gravitational-wave emission for $\gtrsim 25 \mathrm{~ms}$ with fitting factors of 0.92-0.97. This model addresses the two restrictions that prevent matched filtering of postmerger gravitational-wave strain: computational time and poor 
fitting factors. Our model is derived from a hybrid of the two models outlined in Refs. [26,27]. Our model is agnostic to the locations of the frequency peaks and uses Bayesian statistics to determine the actual peak frequencies. Furthermore, the addition of a frequency drift term allows for secular changes in the frequency peak locations. With postmerger signal-to-noise ratios of $\geq 15$, the model can localize the primary postmerger frequency to $\pm_{1.2}^{1.4} \%$ at $95 \%$ confidence, reducing to $\pm_{0.2}^{0.3} \%$ at postmerger signalto-noise ratio of 50. Using the hierarchical model developed in Ref. [28] we can then constrain the tidal parameters and compactness of the progenitor neutron stars. The tidal coupling constant is constrained to $\pm_{12}^{9} \%$ at postmerger signal-to-noise ratios of 15 for a $95 \%$ confidence interval. At postmerger signal-to-noise ratios of 50 this tightens to $\pm 5 \%$.

In Sec. II we outline the model and associated methods used in this paper. In Sec. III we validate the model fits in the time and frequency domains and quantify the goodness of the fits. In Sec. IV we use a Bayes factor detection statistic to determine at what postmerger signal-to-noise ratios a detection occurs and test how the model performs due to uncertainty in the inspiral coalescence time. In Sec. V we calculate posteriors of the dominant postmerger frequency and introduce the hierarchical model from Ref. [28] to find the equation of state parameters for the progenitors. We find constraints on both the tidal coupling constant and the compactness of the progenitors.

\section{METHODOLOGY}

We adopt a model for the postmerger gravitational-wave signal consisting of three exponentially damped sinusoids [26] with additional linear frequency drift terms [27]. The plus, $h_{+}(\boldsymbol{\theta}, t)$, polarization of the gravitational-wave strain is extracted from the right circular polarization, $h(\boldsymbol{\theta}, t)$, as follows:

$$
\begin{aligned}
h(\boldsymbol{\theta}, t) & =h_{+}(\boldsymbol{\theta}, t)-\mathrm{i} h_{\times}(\boldsymbol{\theta}, t) \\
& =\sum_{j=0}^{2} h_{j,+}(\boldsymbol{\theta}, t)-\mathrm{i} h_{j, \times}(\boldsymbol{\theta}, t),
\end{aligned}
$$

$h_{j,+}(\boldsymbol{\theta}, t)=H w_{j} \exp \left[-\frac{t}{T_{j}}\right] \cos \left(2 \pi f_{j} t\left[1+\alpha_{j} t\right]+\psi_{j}\right)$.

Here, $\boldsymbol{\theta}=\left\{H, w_{j}, T_{j}, f_{j}, \alpha_{j}, \psi_{j}: j \in[0,2]\right\}$ are the model parameters where $H$ is the amplitude scaling factor and $w_{j}$ is the relative scaling factor for each mode, $j \in[0,2]$, such that $\sum_{j} w_{j}=1$. The initial frequency of each mode is given by $f_{j}, T_{j}$ are the damping times, $\psi_{j}$ are the initial phases, and $\alpha_{j}$ are the frequency drift terms. The time, $t$, is defined such that $t=0$ corresponds to the coalescence time when the maximum of $h_{+}^{2}(\boldsymbol{\theta}, t)+h_{\times}^{2}(\boldsymbol{\theta}, t)$ occurs, e.g.,
$[16,17,28,29]$. The cross polarization of the $j$ th mode is generated by a $\pi / 2$ phase shift on $h_{j,+}(\boldsymbol{\theta}, t)$. Setting $\alpha_{j}=0$ allows detection of signals corresponding to the crosspolarization model in Ref. [26]. These equations are a subset of the plus polarization model in Ref. [27] with the quadratic drift term set to zero and no explicit modulation of spectral peaks.

We use nine postmerger numerical-relativity simulations from Ref. [30] (see Appendix A for details), selecting only simulations with equal-mass progenitors where a nascent neutron star survives for at least $\sim 25 \mathrm{~ms}$. For equal-mass systems, the tidal parameter of the neutron stars is related to the dimensionless compactness, $C=G M /\left(R c^{2}\right)$, and the second Love number, $k_{2}$, as follows:

$$
\begin{aligned}
\tilde{\Lambda} & =\frac{2}{3} k_{2} C^{-5}, \\
\kappa_{2}^{\mathrm{T}} & =\frac{1}{8} k_{2} C^{-5},
\end{aligned}
$$

where $\tilde{\Lambda}$ is the quadrupolar tidal deformability and $\kappa_{2}^{\mathrm{T}}$ is the total quadrupolar tidal coupling constant. Here, $M$ is the neutron star mass, $R$ is the neutron star radius, $G$ is the gravitational constant, and $c$ is the speed of light. The tidal properties of the progenitors can be estimated from the dominant postmerger frequency using relations found from numerical-relativity simulations with baryonic equations of state [16,18] (although see Refs. [18,31,32] for the consequences of a phase transition to strange matter). We discuss this more in Sec. V.

We inject numerical-relativity waveforms at various postmerger signal-to-noise ratios into a three-detector network (LIGO Hanford, Livingston, and Virgo) at design sensitivity for each interferometer [33,34]. We inject the postmerger signal at a fixed time and fixed sky position, assuming that we know the coalescence time from the inspiral stage. In Sec. IV we test this assumption by determining the uncertainty in the coalescence time for various signal-to-noise ratios. We use the BILBY package [35] with the DYNESTY sampler [36] to sample posteriors, $p(\boldsymbol{\theta} \mid d)$, of the model parameters using the likelihood, $\mathcal{L}(d \mid \boldsymbol{\theta})$, as follows:

$$
\begin{gathered}
p(\boldsymbol{\theta} \mid d)=\frac{\mathcal{L}(d \mid \boldsymbol{\theta}) \pi(\boldsymbol{\theta})}{\mathcal{Z}}, \\
\mathcal{Z}=\int_{\boldsymbol{\theta}} d \boldsymbol{\theta} \mathcal{L}(d \mid \boldsymbol{\theta}) \pi(\boldsymbol{\theta}), \\
\mathcal{L}(d \mid \boldsymbol{\theta}) \propto \exp [-\langle d(t)-h(\boldsymbol{\theta}, t), d(t)-h(\boldsymbol{\theta}, t)\rangle] .
\end{gathered}
$$

Here, $d(t)=s(t)+n(t)$ is the numerical-relativity waveform, $s(t)$, injected into noise, $n(t)$. We simulate ten different Gaussian noise realizations with BILBY, to examine the response of the model to variations in detector noise. We limit this to ten noise realizations to keep the 


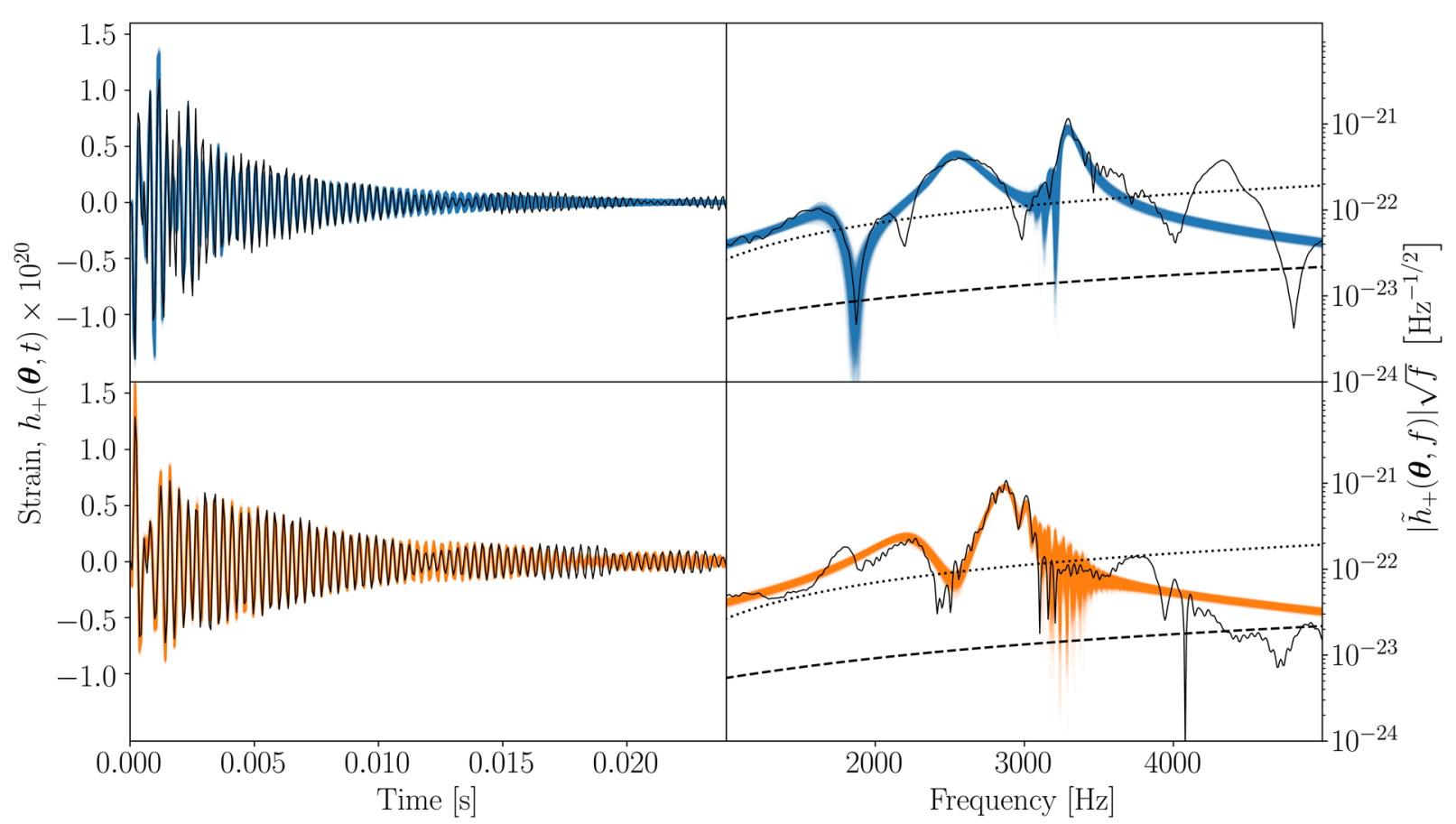

FIG. 1. Waveform reconstruction of numerical-relativity postmerger signal injections. Top panels: time (left) and frequency (right) domain reconstructions of a numerical-relativity simulation using the SLy equation of state with equal mass, $1.35 M_{\odot}$, neutron stars (waveform SLy-M1.350- 4390 ). The postmerger waveform (black curve) is injected at a postmerger signal-to-noise ratio of 50. The reconstructed waveforms are shown in blue. Bottom panels: same as the top panels except the injected waveform is using the LS220 equation of state with equal mass, $1.35 M_{\odot}$, neutron stars (waveform LS220-M1.350- 4684 ). The reconstructed waveforms are shown in orange. Noise sensitivity curves are shown for Advanced LIGO (dashed black) and Advanced Virgo (dotted black) for plots on the right.

computation time manageable. The priors on the model parameters are $\pi(\boldsymbol{\theta})$. The noise-weighted inner product in Eq. (8) is defined by

$$
\left\langle h_{1}, h_{2}\right\rangle \equiv 4 \operatorname{Re} \int d f \frac{\tilde{h}_{1}(f) \tilde{h}_{2}^{*}(f)}{S_{h}(f)},
$$

where $S_{h}$ is the detector's noise power spectral density. We use a sampling frequency of $8192 \mathrm{~Hz}$ to eliminate aliasing of the upper sidebands. We use constrained priors to sort the maximum amplitude for $\tilde{h}_{j,+}(\boldsymbol{\theta}, f)$, such that $\left|\tilde{h}_{j,+}(\boldsymbol{\theta}, f)\right|_{\max }>\left|\tilde{h}_{j+1,+}(\boldsymbol{\theta}, f)\right|_{\max }$. This ensures that the mode zero $(j=0)$ exponentially damped sinusoid corresponds to the dominant postmerger frequency. Full details on the priors are given in Appendix B. The optimal postmerger signal-to-noise ratio, $\rho_{\text {opt }}$, is calculated from the quadrature sum of the optimal postmerger signal-tonoise ratio for each of the three detectors, $\rho_{\mathrm{opt}, i}$ as follows:

$$
\rho_{\mathrm{opt}}^{2}=\sum_{i \in \mathrm{HLV}} \rho_{\mathrm{opt}, i}^{2},
$$

for $t \geq 0$. The matched filter signal-to-noise ratio for a single detector is given by

$$
\rho_{m f}=\frac{\langle d, h(\boldsymbol{\theta})\rangle}{\langle h(\boldsymbol{\theta}), h(\boldsymbol{\theta})\rangle^{\frac{1}{2}}} .
$$

\section{MODEL VALIDATION}

Figure 1 shows the posterior waveforms in the time and frequency domain for the plus polarization of two numerical-relativity postmerger simulations. The two gravitational-wave simulations, SLy-M1.350- 1390 (THC:0036: R03, top) and LS220-M1.350- 4684 (THC:0019:R05, bottom), are injected at a postmerger signal-to-noise ratio of 50. These waveforms are chosen for compatibility with the inferred properties of $\Lambda$ from GW170817 [1,32,37-40]. SLy-M1.350- $\Lambda 390$ is a simulation of equal progenitor mass $1.35 M_{\odot}$ neutron stars with tidal deformability, $\tilde{\Lambda}=$ $390.1\left(\kappa_{2}^{\mathrm{T}}=73.14\right)$ and SLy equation of state. Similarly, LS220-M1.350- $\Lambda 684$ has masses of $1.35 M_{\odot}, \tilde{\Lambda}=683.8$ $\left(\kappa_{2}^{\mathrm{T}}=128.2\right)$ and LS220 equation of state.

We generate posterior waveforms by randomly drawing samples from the posterior distribution $p(\boldsymbol{\theta} \mid d)$. The posterior waveforms are shown as blue (top, SLy-M1.350-

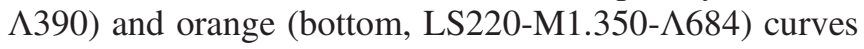
in Fig. 1. The solid black curves show the injected numerical-relativity waveforms. As can be seen in the time-response plots (Fig. 1, left), the posterior samples are tightly clustered around the numerical-relativity simulations, particularly for the first $\sim 15 \mathrm{~ms}$. The phase of waveform SLy-M1.350- $\Lambda 390$ is lost after $\sim 15 \mathrm{~ms}$ (Fig. 1, upper left) though the majority of the spectral content is contained in the first $5 \mathrm{~ms}$ (see Fig. 4). We note 


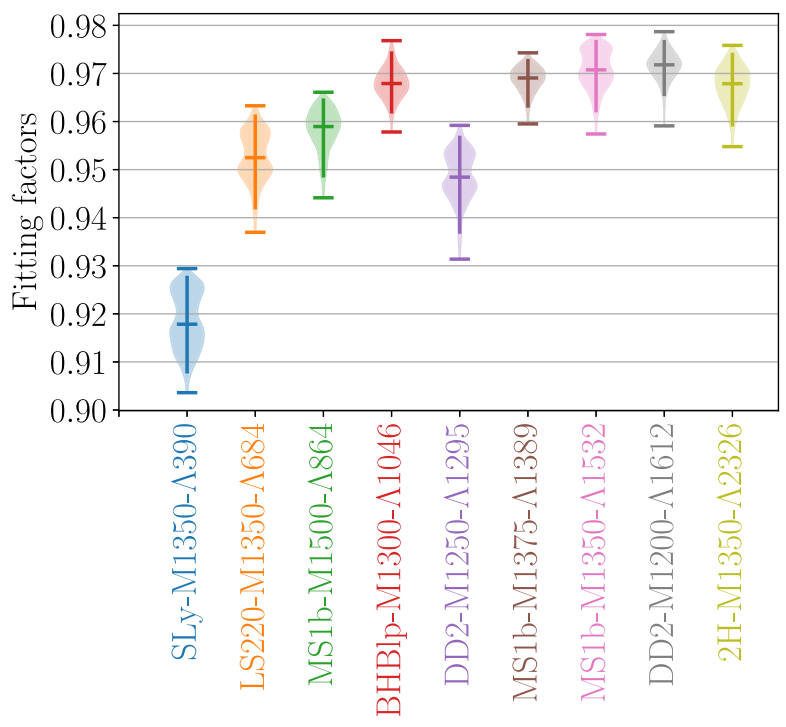

FIG. 2. Fitting-factor distributions, $\mathcal{F}(d(t), h(\boldsymbol{\theta}, t))$, for each postmerger numerical-relativity waveform. The signal-to-noise ratio for the postmerger gravitational-wave strain for each waveform is 50. The upper and lower horizontal bars represent $99.7 \%$ confidence intervals. The central horizontal bar shows the median value. The thick vertical line shows the $95 \%$ confidence intervals. The median fitting factors range between 0.92 to 0.97 which corresponds to a reduction in detection rate from $22 \%$ down to $9 \%$ due to mismatch with the numerical-relativity injections.

that accumulated phase errors in numerical-relativity simulations increase over time.

The frequency-response plots are shown on the right side of Fig. 1, along with the amplitude spectral density of Advanced LIGO (dashed black curve) and Advanced Virgo (dotted black curve) at design sensitivity. The primary frequency peaks are well recovered for both reference waveforms. Two low frequency peaks of SLy-M1.350$\Lambda 390$ are resolved in preference to the upper frequency peak, whereas only one low frequency peak is resolved for LS220-M1.350- $\Lambda 684$. The other two modes are located at the main frequency peak of LS220-M1.350- $\Lambda 684$.

To measure the extent of the waveform mismatch, we calculate the noise-weighted fitting factor between the injected numerical-relativity waveform, $d(t)$, and the posterior waveform, $h(\boldsymbol{\theta}, t)[41]$ :

$$
\mathcal{F}(d(t), h(\boldsymbol{\theta}, t)) \equiv \frac{\langle d(t) \mid h(\boldsymbol{\theta}, t)\rangle}{\sqrt{\langle d(t) \mid d(t)\rangle\langle h(\boldsymbol{\theta}, t) \mid h(\boldsymbol{\theta}, t)\rangle}} .
$$

The fitting factor, calculated with noise from one detector at Advanced LIGO design sensitivity [33], quantifies the loss in signal-to-noise due to signal mismatch in relation to an optimal signal-to-noise ratio, Eq. (10).

The median fitting factors are 0.92 and 0.95, for SLyM1.350- $\Lambda 390$ and LS220-M1.350- $\Lambda 684$, respectively. As the detection rate scales as $\mathcal{F}^{3}$ [41], the reduction in detection rate due to the above mismatch is $22 \%$ and $14 \%$ respectively for these two waveforms.

The fitting factors for all nine numerical-relativity simulations are shown in Fig. 2, with each simulation represented by a different color. Ten different Gaussian noise realizations are used for each numerical-relativity simulation. The $99.7 \%$ confidence intervals for the fitting factors are shown by the upper and lower horizontal bars. The median value is shown by the central horizontal bar, and $95 \%$ confidence intervals are indicated by thick vertical bars. Finally, the distribution of the fitting factors is shown by the width of the shaded areas. The lowest fitting factors, for simulation, SLy-M1.350- $\Lambda 390$, have an average match of 0.92 . Other numerical-relativity injections have fitting factors of $0.95-0.97$. The injection with the softest equation of state underperforms the other injections. This is due to complex dynamics of the nascent neutron star in the first $\sim 2 \mathrm{~ms}$.

\section{SENSITIVITY}

We calculate the Bayes factor between the signal hypothesis and a noise hypothesis to evaluate the sensitivity of our model. We do this by injecting the postmerger signal SLy-M1.350- $\Lambda 390$ into ten different noise realizations at various signal-to-noise ratios. The results are shown in Fig. 3. The distribution of the natural logarithm of the Bayes factor, $\ln (\mathcal{B F})$, is shown for each postmerger signalto-noise ratio along with the $99.7 \%$ confidence intervals (upper and lower horizontal bars) and the median value (middle horizontal bar). We define that strong evidence for

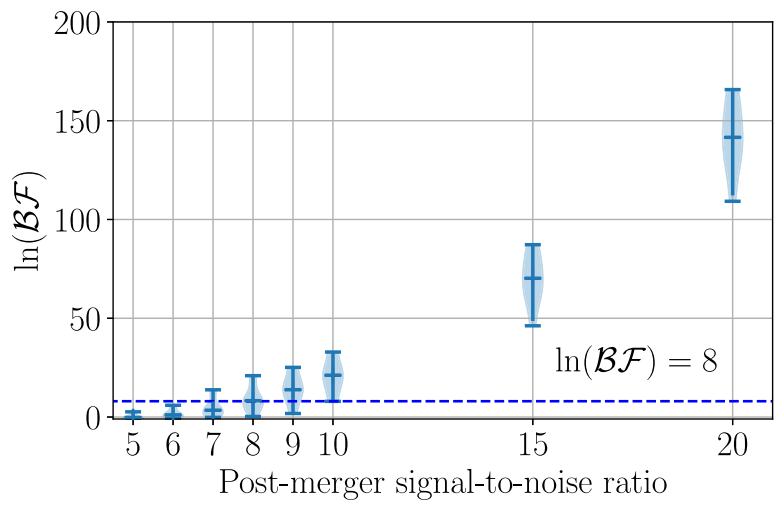

FIG. 3. Natural logarithm of the Bayes factor comparing a signal hypothesis against a noise hypothesis plotted against the postmerger signal-to-noise ratio. The numerical-relativity waveform, SLy-M1.350- 1390 , is injected into ten different noise realizations at the specified signal-to-noise ratio. The upper and lower horizontal bars show the $99.7 \%$ confidence intervals of the $\log$ Bayes factor and the central horizontal bar shows the median value. A postmerger signal-to-noise value of $\gtrsim 10$ is required to ensure strong evidence for a signal hypothesis $[\ln (\mathcal{B F})>8]$ across all ten noise realizations. However, there are some noise realizations where $\ln (\mathcal{B F})>8$ occurs for postmerger signal-tonoise ratios as low as 7 . 


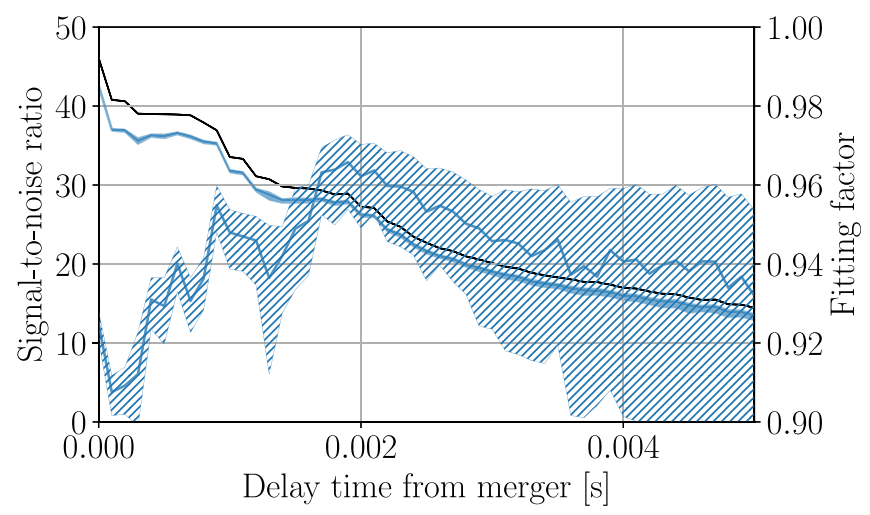

FIG. 4. Variation of fitting factor (hatched blue) and matched filter signal-to-noise ratio (solid blue) for differing values of the delay time after the time of coalescence. The shaded regions show $95 \%$ confidence intervals. The optimal signal-to-noise ratio is also shown (solid black). Although the fitting factor is lower when the entire postmerger signal is used, the matched filter signal-to-noise ratio is largest. The fitting factor is lower for smaller delay times due to the complex dynamics of the nascent neutron star.

a signal hypothesis over a noise hypothesis corresponds to a Bayes factor exceeding $3000(\ln (\mathcal{B F})>8.0)$, e.g., [42]. In this case a signal hypothesis is 3000 times more likely than a noise hypothesis. This occurs with postmerger signal-to-noise ratios of $\gtrsim 10$ for all tested noise realizations. There are some noise realizations where the Bayes factor threshold is exceeded for postmerger signal-to-noise ratios of $\approx 7-9$.

An important consideration for our signal model is the uncertainty in the coalescence time as measured from the gravitational-wave inspiral signal. This determines how close we can get to the true coalescence time for the binary neutron star merger. In Fig. 4 we investigate the model performance to uncertainties in the coalescence time. We show how the fitting factor and matched-filter signalto-noise ratio change when starting the adopted model at various times after the coalescence time. We multiply the numerical-relativity injection, $d(t)$, by the Heaviside step function, $\mathcal{H}\left(t-t_{\text {delay }}\right)$, and evaluate the model, $h\left(\boldsymbol{\theta}, t-t_{\text {delay }}\right)$, for $t \geq t_{\text {delay }}$. The matched filter signal-to-noise ratio is calculated using Eq. (11) with a single detector at Advanced LIGO sensitivity. We use numerical-relativity injection, SLy-M1.350- $\Lambda 390$, selected due to compatibility with the tidal parameters inferred from GW170817. A delay time of zero includes the entire postmerger waveform, whereas a delay time of $2 \mathrm{~ms}$ excludes the first $2 \mathrm{~ms}$ of the injection after the coalescence time. The fitting factor is lower $(\sim 0.91)$ for small delay times and increases to $\sim 0.96$ at $2 \mathrm{~ms}$. The fitting factor is lower in the first $2 \mathrm{~ms}$ due to complex dynamics of the nascent neutron star. In Fig. 4, the matchedfilter signal-to-noise ratio is almost monotonically decreasing as expected. Even though the fitting factors are lower at zero delay time, the matched-filter signal-to-noise ratio is at

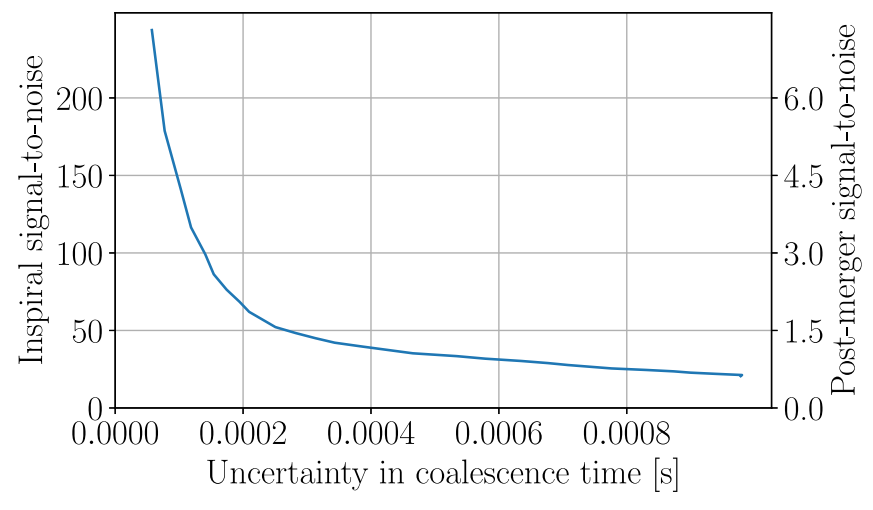

FIG. 5. Uncertainty in coalescence time plotted with signal-tonoise ratios. The coalescence time uncertainty has been determined by a Fisher matrix approximation. The left axis is the signal-to-noise ratio for a two detector network of Advanced LIGO at design sensitivity for a binary neutron star inspiral. The right axis is the postmerger signal-to-noise ratio for a three detector network of Advanced LIGO and Advanced Virgo at design sensitivity using numerical-relativity simulation SLyM1.350- 4390 . Postmerger signal-to-noise ratios above 6.0 have coalescence time uncertainties of less than $0.1 \mathrm{~ms}$.

maximum. Therefore, from a sensitivity perspective, a minimum delay time is preferred.

To estimate the uncertainties of the time of coalescence of the inspiral signal as a function of the signal-to-noise ratio of the inspiral signal, we use a Fisher matrix approximation. We assume that the signal parameters $\vartheta$ follow a Gaussian distribution:

$$
p(\Delta \boldsymbol{\vartheta}) \propto \exp \left[-\frac{1}{2} \Gamma_{i j} \Delta \vartheta^{i} \Delta \vartheta^{j}\right]
$$

Here, $\Delta \vartheta_{i}=\vartheta^{i}-\hat{\vartheta}^{i}, \hat{\vartheta}^{i}$ are the best fit inspiral parameters and $\Gamma_{i j}=\left(\partial h / \partial \vartheta_{i} \mid \partial h / \partial \vartheta_{j}\right)$ is the expected Fisher information matrix. The estimated errors of the parameters, $\vartheta_{i}$, are obtained by taking the diagonal elements of the Fisher information matrix. The relevant parameters within our approximation are $\boldsymbol{\vartheta}=\left(\mathcal{M}, q, \phi_{c}, \tilde{\Lambda}, t_{c}, H\right)$, where $\mathcal{M}$ is the chirp mass, $q$ is the mass ratio, and $\phi_{c}$ is the phase of coalescence. The average-weighted tidal deformability is $\tilde{\Lambda}$, $t_{c}$ is the time of coalescence and $H$ is the amplitude of the inspiral waveform. We calculate the errors on $\vartheta_{i}$ assuming an equal mass $1.4 M_{\odot}$ nonrotating progenitor system. The expected uncertainties for the coalescence time are shown in Fig. 5. The left axis shows the inspiral signal-to-noise ratio for an optimally oriented source into a two detector LIGO network at design sensitivity. We use Fig. 8 from Ref. [43] to determine the luminosity distance, $D_{L}$, from the inspiral signal-to-noise ratio. We calculate the product of $D_{L 0} \approx$ $475 \mathrm{Mpc}$ (at $z \approx 0.1$ ) with the corresponding inspiral signalto-noise ratio, $\rho_{\text {inspiral }, 0} \approx 7$. We inject the numericalrelativity postmerger waveform, SLy-M1.350- $\Lambda 390$, at luminosity distance, $D_{L}=D_{L 0}\left(\rho_{\text {inspiral }, 0} / \rho_{\text {inspiral }}\right)$, and 
evaluate the postmerger signal-to-noise ratio using the Advanced LIGO and Virgo detector network at design sensitivity. The right axis in Fig. 5 shows the corresponding postmerger signal-to-noise ratio.

For postmerger signal-to-noise ratios larger than 6 , the uncertainty in the coalescence time is less than $0.1 \mathrm{~ms}$. This shows that, for postmerger signal-to-noise ratios of interest in this work, the coalescence time is similarly constrained. The uncertainty in coalescence time can be related to Fig. 4 to show that the resultant matched filter signal-to-noise ratio is not significantly reduced due to the uncertainty in the coalescence time.

\section{PARAMETER ESTIMATION}

Estimation of the primary postmerger frequency is another important indicator of the model performance. We estimate this by calculating posteriors of the peak frequency, $f_{\text {peak }}$, of the dominant mode. Figure 6 shows posteriors of $f_{\text {peak }}$ as a function of postmerger signal-tonoise ratio. These have been calculated for an injection of SLy-M1.350- $\Lambda 390$ at postmerger signal-to-noise ratios of $\geq 9$. The noise realization was kept the same for all injections. Blue shading indicates regions of 95\% confidence intervals and the median values are shown as blue dots. The frequency corresponding to the maximum value of the characteristic strain spectrum of the numericalrelativity signal, $\left|\tilde{s}_{+}(f)\right| \sqrt{f}$, is shown as a black horizontal line. This can be thought of as an approximation of the true injected value of $f_{\text {peak }}$. The $f_{\text {peak }}$ frequency is constrained within $95 \%$ confidence intervals to $3310 \pm_{38}^{46} \mathrm{~Hz}$ at a postmerger signal-to-noise ratio of 15 which corresponds to $\pm_{1.2}^{1.4} \%$. At a postmerger signal-to-noise ratio of 50 , the precision increases to $3296 \pm_{8}^{11} \mathrm{~Hz}\left( \pm_{0.2}^{0.3} \%\right)$. The posteriors for $f_{0}, \alpha_{0}, f_{1}$ and $\alpha_{1}$, determined for all numericalrelativity injections at a postmerger signal-to-noise ratio of 50, are shown in Figs. 9-17 in Appendix C.

We also analyze injections of SLy-M1.350- 390 using BAYESWAVE $[44,45]$. BAYESWAVE uses a variable number of Morlet-Gabor wavelets to model the signal, where both the number and the properties of the wavelets are marginalized over. This is an established method for postmerger studies [2,40]. References [46,47] have performed simulations using BAYESWAVE to infer the postmerger properties of binary neutron star mergers. We compute the posteriors of the spectral frequency peak, $f_{\text {peak }}$, using BAYESWAVE following Ref. [46]. Here, $f_{\text {peak }}$, the frequency of the highest peak in the Fourier power spectrum of the signal, is determined for each sample from the BAYESWAVE posterior. For samples that do not have a peak, $f_{\text {peak }}$ is computed using random draws from its prior [46]. Figure 6 shows the $95 \%$ confidence intervals of $f_{\text {peak }}$ for each postmerger signal-to-noise ratio in brown. The median values are shown as brown crosses. The BAYESWAVE frequency posteriors are consistent with Refs. [46,47].

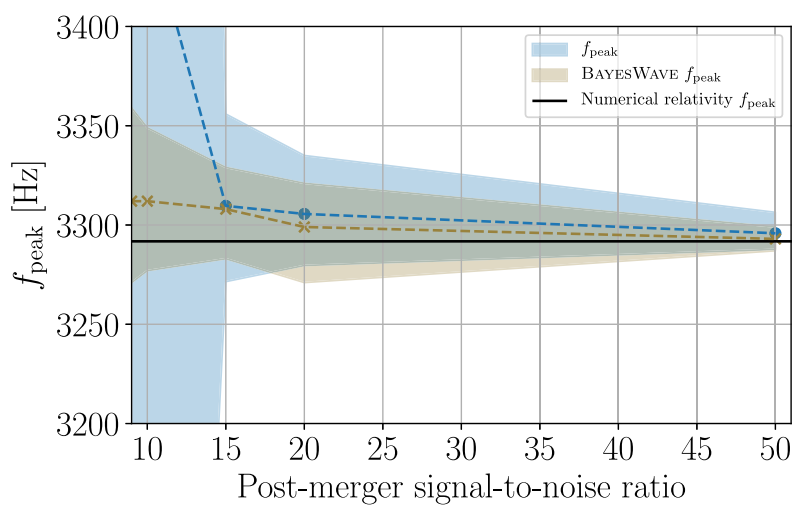

FIG. 6. Primary postmerger frequency comparison between our model (blue) and BAYESWAVE (brown). The posteriors are plotted against the postmerger signal-to-noise ratio for injection SLyM1.350- $\Lambda 390$. The 95\% confidence intervals are shaded. The median points are shown as blue dots and brown crosses, for our model and BAYESWAVE, respectively. The frequency corresponding to the peak of the spectral response of the injection is also shown (solid black line).

The posteriors for $f_{\text {peak }}$ are similarly constrained for both BAYESWAVE and our model for postmerger signal-to-noise ratios of $\gtrsim 20$. BAYESWAVE is more constrained for postmerger signal-to-noise ratios of $\sim 9-15$. Both methods are able to recover the injected dominant postmerger frequency. BAYESWAVE can generate very high fitting factors; the fitting factors for SLy-M1.350- $\Lambda 390$ at a postmerger signal-to-noise ratio of 50 are $\approx 0.99$. The dimensionality of BAYESWAVE is $\sim 90$ ( $\sim 18$ wavelets) at this postmerger signal-to-noise ratio. The dimensionality of our adopted model is 15 with fitting factors of $\approx 0.92$ for SLy-M1.3501390. Furthermore, BAYESWAVE can generalize to any signal (e.g., glitches). In contrast, our model has been developed to suit a postmerger gravitational-wave signal. The parameters in our model are interpretable: for example, in Fig. 9, the $\alpha_{0}$ value for SLy-M1.350- $\Lambda 390$ is $-1.60 \pm_{0.26}^{0.50}$ which shows that the frequency of the dominant gravitational-wave mode is decreasing.

The hierarchical model from Ref. [28] allows a bidirectional relationship between equal mass progenitor neutron star properties $\left(C, M, \kappa_{2}^{\mathrm{T}}\right)$ and numericalrelativity postmerger simulations. This is achieved by a two step process. First, the progenitor properties are used to solve $\bar{C}\left(M, \kappa_{2}^{\mathrm{T}}\right)=C$ using a power-law relationship. Second, the model parameters, $\boldsymbol{\Theta}$, are determined by solving $h_{c}=\boldsymbol{\Theta} \boldsymbol{X}\left(\bar{C}\left(M, \kappa_{2}^{\mathrm{T}}\right), M, \kappa_{2}^{\mathrm{T}}\right)$, where $h_{c}$ is the numerical-relativity amplitude spectra for the characteristic strain $\left(h_{c}(f)=|\tilde{h}(f)| \sqrt{f}\right)$. Here, $\boldsymbol{X}\left(\bar{C}\left(M, \kappa_{2}^{\mathrm{T}}\right), M, \kappa_{2}^{\mathrm{T}}\right)$ is a design matrix derived from the progenitor properties $M$ and $\kappa_{2}^{\mathrm{T}}$.

We use the posteriors from Sec. III to calculate the amplitude of the characteristic spectrum $\left|\tilde{h}_{+}(\boldsymbol{\theta}, f)\right| \sqrt{f}$ and use the trained model, $\boldsymbol{\Theta}$, to determine the hierarchical model posteriors on $\kappa_{2}^{\mathrm{T}}$ and $C$. The cross-polarization 


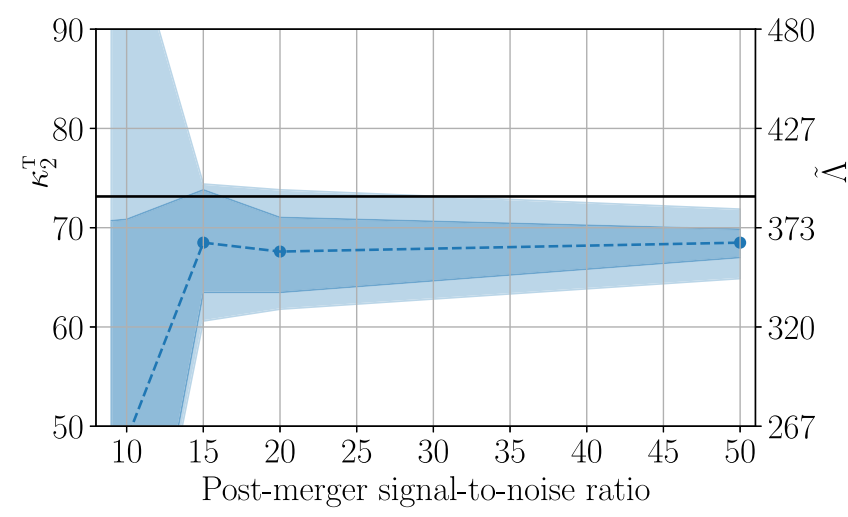

FIG. 7. Tidal coupling constant posteriors versus postmerger signal-to-noise ratio for numerical-relativity waveform SLyM1.350- $\Lambda 390$. The tidal coupling constant is inferred from the hierarchical model [28] using the magnitude of the posterior waveforms, $\left|h_{+}(\boldsymbol{\theta}, t)\right|$. The $68 \%$ (dark blue) and $95 \%$ (light blue) confidence intervals are shown along with the median values (blue dots). The true value for $\kappa_{2}^{\mathrm{T}}$ is shown as the solid horizontal line. The corresponding tidal deformability values are shown on the secondary vertical axis.

waveforms are discarded because the hierarchical model only uses the magnitude of the spectra, and $\left|\tilde{h}_{+}(\boldsymbol{\theta}, f)\right|=$ $\left|\tilde{h}_{\times}(\boldsymbol{\theta}, f)\right|$. The hierarchical model, $\boldsymbol{\Theta}$, was previously trained on 35 numerical-relativity simulations from Ref. [17], a distinct set of numerical-relativity simulations to those used in this paper. Therefore, this is an out-ofsample model validation.

Figure 7 shows the inferred posteriors for $\kappa_{2}^{\mathrm{T}}$ with $68 \%$ and $95 \%$ confidence intervals in dark blue and light blue respectively. The true injected value of $\kappa_{2}^{\mathrm{T}}$ is shown by the horizontal solid black line and the median values as blue dots. The vertical axis shows both the quadrupolar tidal coupling constant (left axis) and the quadrupolar tidal deformability (right axis). The values inferred for the tidal parameters of the progenitor neutron stars are lower than the true value of the numerical-relativity injection, though the $95 \%$ confidence interval excludes the true value only at a postmerger signalto-noise ratio of 50. The tidal coupling constant at a postmerger signal-to-noise ratio of 15 is constrained at $95 \%$ confidence intervals to $68.5_{-7.9}^{+5.9}$, which tightens to $68.5_{-3.6}^{+3.4}$ for a postmerger signal-to-noise ratio of 50 .

There are a number of factors that will impact on the performance of the hierarchical model. First, the numericalrelativity spectra from Ref. [17], which were used in Ref. [28] to train the model, are a distinct set of simulations to those in use in this paper [30]. Specifically, waveform SLy-M1.350- $\Lambda 390$ is available in both sets of numericalrelativity simulations (Refs. [17,30]), and, although the primary postmerger peak occurs at the same frequency, the spectral response for the other frequencies are different (fitting factor $=0.90$ ). Second, the simulation outputs can be dependent on the spatial and temporal resolution, which can lead to waveform changes related to parameters like

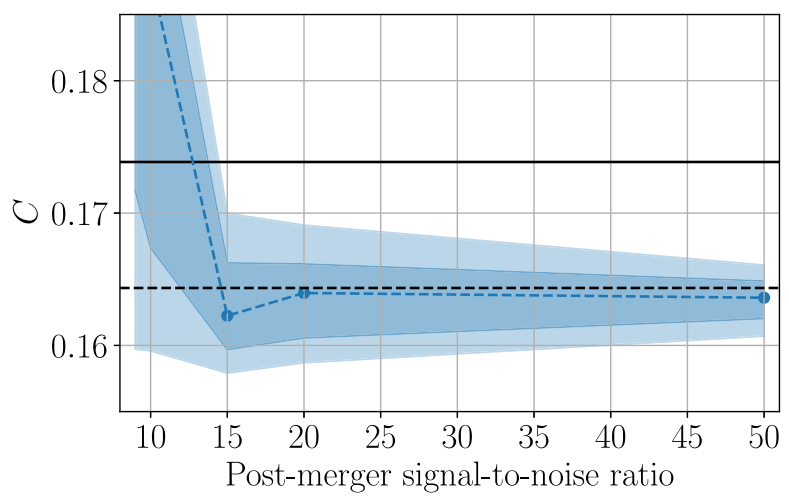

FIG. 8. Compactness posteriors versus postmerger signal-tonoise ratio for numerical-relativity waveform SLy-M1.350- $\Lambda 390$. The $68 \%$ (dark blue) and 95\% (light blue) confidence regions are shown. The compactness is inferred from the hierarchical model [28]. The compactness inferred directly from the numericalrelativity waveform (dashed black line) and the compactness of the progenitor neutron stars (solid black line) are also shown. See the text for an explanation of the offset in these two values.

collapse time, primary oscillation frequency and decay time constants. To ensure the performance of the hierarchical model, the simulations used in the training set and test set should be consistent and not contradictory. Third, the hierarchical model is an approximate model, and was trained on 35 compatible waveforms; (for details, see Ref. [28]). Given the small training set, the hierarchical model results are consistent. The complexity of the model could be increased as additional consistent numericalrelativity simulations become available.

Hierarchical model posteriors for the compactness, $C$, are shown in Fig. 8. The $68 \%$ and $95 \%$ confidence intervals are shaded dark blue and light blue respectively, and the median values are shown with blue dots. The true value corresponding to the injected numerical-relativity simulation is shown as a horizontal solid black line. The value inferred from the numerical-relativity simulation using the hierarchical model is shown as a horizontal black dashed line. The hierarchical posteriors for $C$ are clustered around the value inferred directly from the hierarchical model for the numerical-relativity simulation. This is expected and due to the mismatch between the numerical-relativity injection and the set of numerical-relativity simulations used to train the hierarchical model. The compactness has been constrained to $0.162_{-0.004}^{+0.007}$ at postmerger signal-tonoise ratios of 15 tightening to $0.164_{-0.003}^{+0.002}$ at signal-tonoise ratios of 50 to $95 \%$ confidence intervals. The posteriors for the compactness, $C$, only narrow moderately as the postmerger signal-to-noise ratio is increased.

\section{DISCUSSION}

We use an analytical model to characterize gravitationalwave strain from nine numerical-relativity simulations 
selected such that the postmerger oscillations persist for $\sim 25 \mathrm{~ms}$. The median noise-weighted fitting factors for the posterior waveforms range between $0.92-0.97$ for injections with postmerger signal-to-noise ratios of 50. This corresponds to a loss in detection rate of $22 \%-12 \%$ when compared to a signal without mismatch. We measure the Bayes factor in favor of signal detection with numericalrelativity simulation SLy-M1.350- $\Lambda 390$ and find that successful detections occur with postmerger signal-to-noise ratios of $\geq 10$ with possible detections as low as postmerger signal-to-noise ratios of 7 , depending on the specific noise realization. This indicates that this model could be used for parameter estimation and detection if a postmerger signal louder than signal-to-noise ratio of 10 was coincident with an inspiral detection. We find that this corresponds to a distance of $\sim 10 \mathrm{Mpc}$ for an optimally oriented system using a three-detector network (LIGO Hanford, Livingston, and Virgo) at design sensitivity.

We determine that starting the model at the time of coalescence results in the maximum matched filter signalto-noise ratio even though the fitting factors are lower in the vicinity of the merger due to the dynamics of the nascent neutron star. We find that the uncertainty in the time of coalescence for the inspiral of the progenitor neutron stars is less that $0.1 \mathrm{~ms}$ for a postmerger signal-to-noise ratio of $\geq 6$ and show that this corresponds to a maximum matchedfilter signal-to-noise ratio.

The gravitational-wave strain of the inspiral can constrain the equation of state for the cold neutron star at the high inspiral signal-to-noise ratios $(\gtrsim 200)$ required for postmerger detection of the remnant (see Fig. 5). This can place additional constraints on the priors for the dominant postmerger frequency. However, a phase transition in the hot postmerger remnant [18,31,32], and uncertainty in the numerical-relativity calculations due to computational trade-offs, may result in a postmerger gravitational-wave signal that is quantitatively different than the numericalrelativity simulations. With this in mind, we assume a more general, agnostic set of priors (see Appendix B).

Using numerical-relativity waveform SLy-M1.350$\Lambda 390$, selected for its compatibility with $\Lambda$ values determined from GW170817, e.g., [1,32,37-40], we constrain the primary postmerger frequency to a range of $3310 \mathrm{~Hz} \pm_{1.2}^{1.4} \%$ for $95 \%$ confidence intervals at postmerger signal-to-noise ratios of 15 . The precision increases to $3296 \mathrm{~Hz} \pm_{0.2}^{0.3} \%$ for postmerger signal-tonoise ratios of 50 .

We show that our model and BAYESWAVE similarly constrain the dominant postmerger frequency, $f_{\text {peak }}$, for postmerger signal-to-noise ratios of $\gtrsim 20$. For postmerger signal-to-noise ratio of $\sim 9-15$ BAYESWAVE is better able to constrain $f_{\text {peak }}$. We generate fitting factors of $\approx 0.99$ using BAYESWAVE for SLy-M1.350- $\Lambda 390$ at a postmerger signalto-noise ratio of 50 . The corresponding fitting factors from our model are $\approx 0.92$. The dimensionality of the
BAYESWAVE posterior reconstruction is significantly larger than our analytic; 90 dimensions for BAYESWAVE cf. 15 for ours. Moreover, our adopted model is interpretable and can supply additional information about the individual modes (e.g., frequency drifts and exponential damping time constants).

We use the hierarchical model from Ref. [28], which has been trained on numerical-relativity waveforms from Ref. [17], to determine posteriors for $\kappa_{2}^{\mathrm{T}}$ and $C$. We obtain $95 \%$ confidence intervals on $\kappa_{2}^{\mathrm{T}}$ (and $\tilde{\Lambda}$ ) of $\pm_{12}^{9} \%$ at a postmerger signal-to-noise ratio of 15 with increasing precision to $\pm 5 \%$ at a postmerger signal-to-noise ratio of 50. The $95 \%$ confidence intervals on $C$ range from $\pm_{2.7}^{4.3} \%$ at postmerger signal-to-noise ratios of 15 to $\pm_{1.8}^{1.5} \%$ at postmerger signal-to-noise ratios of 50. However, the injected value for $C$ is outside the $95 \%$ confidence interval.

It should be noted that the inferred posteriors for $C$ are centered around the compactness value inferred directly from the numerical-relativity simulation. This indicates that the offset in the inferred compactness values is caused by the difference in the SLy numerical-relativity simulations between Ref. [17] and Refs. [48,49]. Contradictory training waveforms will increase uncertainties in the hierarchical model. The 35 numerical-relativity simulations used to train the hierarchical model were homogeneous, changing only the equation of state and the progenitor masses between simulations, keeping other simulation parameters the same. It should also be emphasized that, because the numerical-relativity simulations are drawn from independent sources, the posteriors of $\kappa_{2}^{\mathrm{T}}(\tilde{\Lambda})$ and $C$ are true out-ofsample estimates. We expect the estimates of $C$ and $\kappa_{2}^{\mathrm{T}}$ to become more consistent with the injected value as the training set is increased in size and covers more system and progenitor properties.

In addition to the aforementioned analytical models $[26,27]$, other works have generated analytical postmerger gravitational-wave models. In Ref. [11], a model was generated for the time-based amplitude and phase of the complex gravitational-wave strain using a smooth piecewise function for the amplitude. The time-based phase was fit by the combination of a polynomial and exponentially damped sinusoid using an iterative covariance matrix adaption evolution search fitting algorithm. The maximum fitting factors were calculated in the time domain without noise weighting and are not directly comparable to the noise-weighted fitting factors calculated with Eq. (9). The maximum fitting factors were $\sim 0.92-0.98$ for $95 \%$ of the 54 waveforms.

A frequency-domain model was introduced in Ref. [50] from analyzing the major spectral peaks of the whitened power spectrum. The power of the dominant postmerger frequency peak was estimated by a trapezoidal structure and the model parameters were determined with a leastsquares algorithm. No fitting factors were calculated in this reference, as the goal was estimating source redshifts. 
This model was extended in Ref. [16] to add a Gaussian component to the fundamental postmerger frequency using a nonlinear least-squares fit. The goal of the fits in Ref. [16] were qualitative, rather than quantitative and no fitting factors were stated.

The model used in Ref. [26] consists of three exponentially damped sinusoids centred at frequencies $\left(f_{2-0}, f_{\text {spiral }}, f_{\text {peak }}\right)$ which are described in Section I. In contrast, the model introduced in Ref. [27], consists of two exponentially damped sinusoids, the first centred on $f_{1}$ which is modulated by frequency $f_{1 e}$, and the second is centred on the dominant postmerger frequency, $f_{2}$, with a linear and quadratic frequency drift terms. This model produced fits of $\sim 80-94 \%$. In Ref. [51], a frequencydomain model was developed for a single dampedsinusoid. This model was based on three or six parameters and used Bayesian inference to estimate the parameters for 120 numerical-relativity simulations. They obtained fitting factors of $\sim 0.60-0.98$. Reference [19] parameterised the instantaneous amplitude and phase of the time-based gravitational-wave strain using 172 numerical-relativity simulations. Their model uses a rationalpolynomial fit based on the progenitor properties $\left(M_{1}\right.$, $M_{2}, \quad \kappa_{2}^{\mathrm{T}}$ ) derived in Refs. [14,52,53]. They achieved fitting factors of $\sim 0.30-0.85$ in zero noise.

The fitting factors obtained in our paper compare favorably to those listed above; our maximum fitting factors are above 0.93 for all waveforms, cf. [11], and our minimum fitting factors are above 0.90 across all waveforms, cf. [19,51]. The fitting factor is more sensitive to deviations in the time-based phase or Fourier phase response, than it is to amplitude deviations. The fits in Ref. [19] could possibly be improved by adding in more flexibility in the phase response. Our model bypasses the phase matching difficulty by directly fitting the phase with parameters, $\left(f_{j}, \alpha_{j}, \psi_{j}\right)$, from the injected signals from all three interferometers. Although Ref. [51] does directly fit the phase, the first-order model is too restricted to obtain higher fitting factors and better results may be obtained by increasing the order of the model. The number of numerical-relativity simulations used in Refs. $[11,19,51]$ was significantly larger than the nine simulations we use here, including additional mass ratios, spin configurations and eccentricity values. We leave it for future work to expand the number of numericalrelativity simulations to test this model.

Although numerical-relativity simulations currently provide the best estimate of the postmerger gravitational-wave strain, future postmerger signals may not be consistent with these state-of-the-art simulations. With this in mind, our model matches the numericalrelativity simulations well, but it is more flexible than these simulations. This is important because this method is a middle ground between simulations of known waveforms, and more general (e.g., unmodeled excess power and BAYESWAVE) methods. Nevertheless, numerical-relativity simulations are the primary method of investigating the dynamical physics of the postmerger region and research into these simulations is vital.

\section{ACKNOWLEDGMENTS}

P. D. L. is supported through Australian Research Council (ARC) Future Fellowship No. FT160100112, ARC Discovery Project No. DP180103155, and ARC Centre of Excellence No. CE170100004. A. R. C. is supported by ARC Grant No. DE190100656. S. G. and J. A. C. gratefully acknowledge the NSF for financial support from Grants No. PHY 1806580, No. PHY 1809572, and No. TGPHY120016. The Flatiron Institute is supported by the Simons Foundation. This research has made use of data, software and/or web tools obtained from the Gravitational Wave Open Science Center [54], a service of LIGO Laboratory, the LIGO Scientific Collaboration and the Virgo Collaboration. LIGO is funded by the U.S. National Science Foundation. Virgo is funded by the French Centre National de Recherche Scientifique (CNRS), the Italian Istituto Nazionale della Fisica Nucleare (INFN) and the Dutch Nikhef, with contributions by Polish and Hungarian institutes. The authors are grateful for computational resources provided by the LIGO Laboratory and supported by National Science Foundation Grants No. PHY-0757058 and No. PHY-0823459. This research was done using resources provided by the Open Science Grid [55,56], which is supported by the National Science Foundation Award No. 1148698, and the U.S. Department of Energy's Office of Science. We are grateful to Sukanta Bose for valuable comments on the manuscript.

\section{APPENDIX A: NUMERICAL RELATIVITY SIMULATIONS}

We use nine simulations from the CORE gravitational wave database [30] for binary neutron star mergers. The simulations are listed by their equation of state, the

TABLE I. Numerical relativity simulations.

\begin{tabular}{lcc}
\hline \hline Designator & Simulation name & Citations [30] \\
\hline SLy-M1.350- $\Lambda$ 390 & THC:0036:R03 & {$[49]$} \\
LS220-M1.350- $\Lambda 684$ & THC:0019:R05 & {$[57]$} \\
MS1b-M1.500- $\Lambda 864$ & BAM:0088:R01 & $\ldots$ \\
BHBlp-M1.300- $\Lambda 1046$ & THC:0002:R01 & {$[38,58]$} \\
DD2-M1.250- $\Lambda 1295$ & THC:0011:R01 & {$[38,58]$} \\
MS1b-M1.375- $\Lambda 1389$ & BAM:0070:R01 & {$[48]$} \\
MS1b-M1.350- $\Lambda 1532$ & BAM:0065:R03 & {$[12]$} \\
DD2-M1.200- $\Lambda 1612$ & THC:0010:R01 & {$[38,58]$} \\
2H-M1.350- $\Lambda 2326$ & BAM:0002:R02 & {$[12]$} \\
\hline \hline
\end{tabular}


progenitor mass, and the quadrupolar tidal deformability. We limit our simulations to those with equal-mass progenitors for compatibility with the hierarchical model in Ref. [28]. We choose simulations with the highest resolution such that the remnant was transmitting gravitational waves for $\sim 25 \mathrm{~ms}$. In some cases increasing the resolution resulted in a reduced lifetime of the remnant. Table I shows the simulation designator for this paper, the name of the waveform in the CORE database, and the citation for the associated simulation in the metadata (if available).

\section{APPENDIX B: PRIORS}

The priors are listed in Eqs. (B1)-(B8) with $\mathcal{U}(a, b)$ representing a uniform prior distribution from $a$ to $b$. The mode number $j$ is limited to $\{0,1,2\}$ and the mode number $i$ is restricted to $\{0,1\}$. The priors in Eqs. (B7) and (B8) are constrained priors. These restrictions are enforced in addition to the standard priors. The prior in Eq. (B7) ensures that the maximum spectral amplitude of each mode is decreasing. This results in $f_{0}$ converging to the loudest peak:

$$
\begin{gathered}
\log _{10} H \sim \mathcal{U}(-24,-19) \\
f_{j} \sim \mathcal{U}(1000,5000) \\
\log _{10} T_{j} \sim \mathcal{U}(-4.0,0.3) \\
\psi_{j} \sim \mathcal{U}(-\pi, \pi) \\
\alpha_{j} \sim \mathcal{U}(-6.4,6.4) \\
w_{i} \sim \mathcal{U}(0.0,1.0) \\
\log _{10}\left(\frac{\max \left|\tilde{h}_{j}(f)\right|_{f}}{\max \left|\tilde{h}_{j+1}(f)\right|_{f}}\right) \sim \mathcal{U}(0.0,10.0) \\
w_{0}+w_{1} \sim \mathcal{U}(0.0,1.0)
\end{gathered}
$$

$w_{2}$ is calculated as

$$
w_{2}=1-w_{0}-w_{1}
$$

ensuring that $\sum_{j} w_{j}=1$ and $w_{2} \in[0,1]$ as required.

\section{APPENDIX C: POSTERIORS FOR ALL NUMERICAL-RELATIVITY INJECTIONS}

Selected posteriors for all numerical-relativity simulations are shown in Figs. 9-17. The waveforms are injected at a postmerger signal-to-noise ratio of 50 . The posteriors shown are $f_{0}, \alpha_{0}, f_{1}$ and $\alpha_{1}$. The posteriors are colored as per Fig. 2. The frequency drift term, $\alpha_{0}$, for the dominant postmerger frequency is negative for some simulations. Most numerical-relativity simulation spectrograms show that the dominant frequency is constant or increases slightly over time, however, many simulations do show decreasing evolution of the dominant postmerger oscillation frequency, e.g., [58-60].

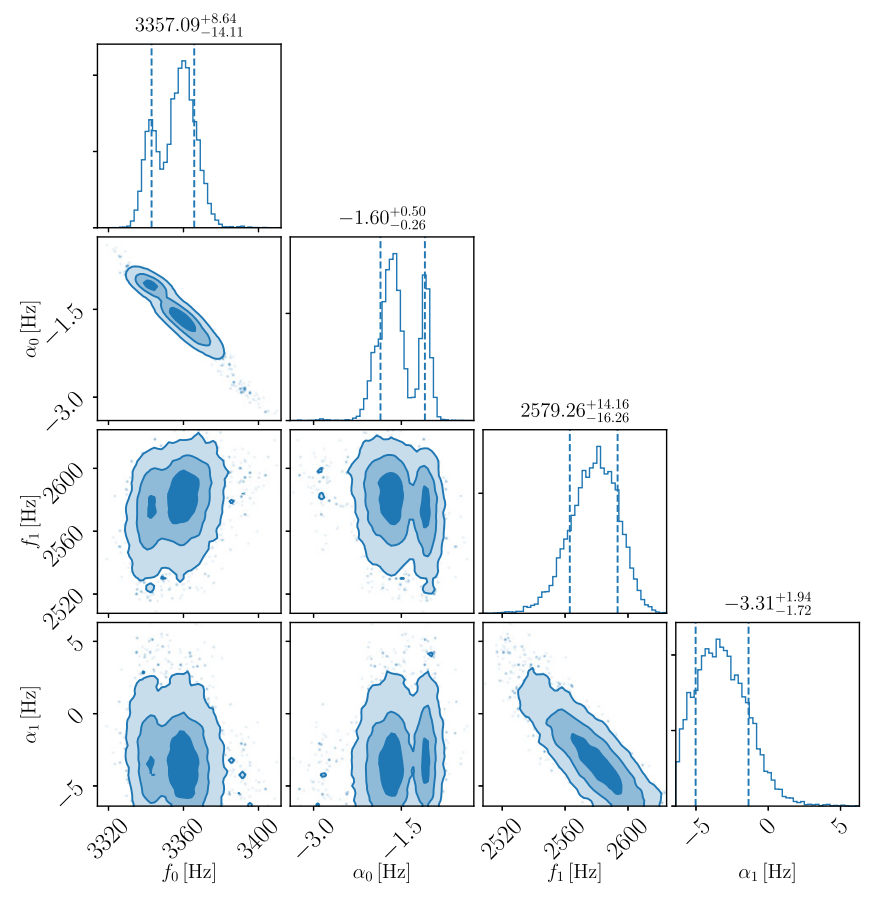

FIG. 9. Selected posteriors for numerical-relativity postmerger injection using the equation of state SLy with equal mass, $1.35 M_{\odot}$, neutron stars (waveform SLy-M1.350- 1390 ). The numerical-relativity simulation was injected at a postmerger signal-to-noise ratio of 50 . 


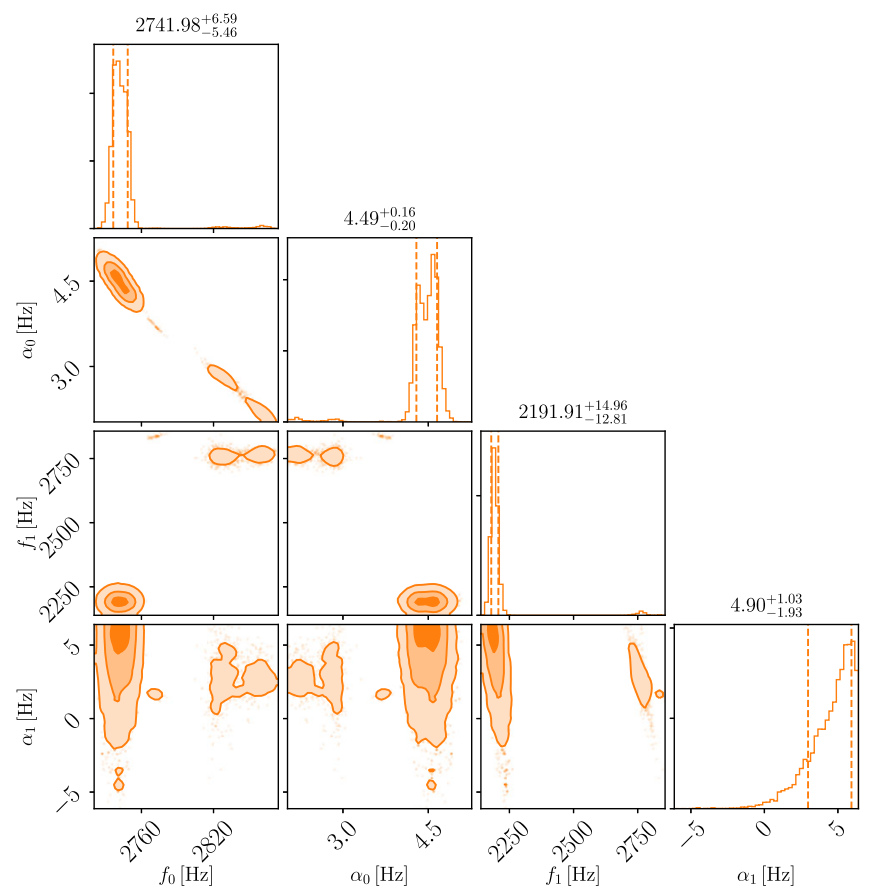

FIG. 10. As per Fig. 9 using the equation of state LS220 with equal mass, $1.35 M_{\odot}$, neutron stars (waveform LS220-M1.350-

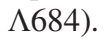

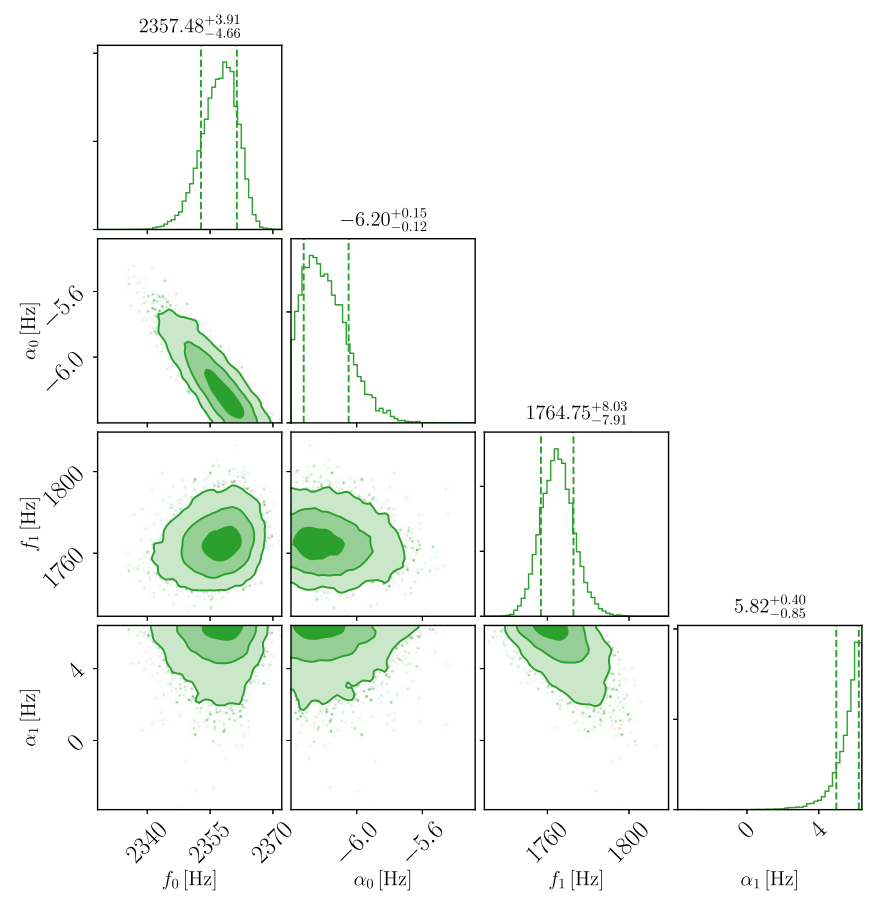

FIG. 11. As per Fig. 9 using the equation of state MS1b with equal mass, $1.50 M_{\odot}$, neutron stars (waveform MS1b-M1.500-

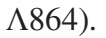

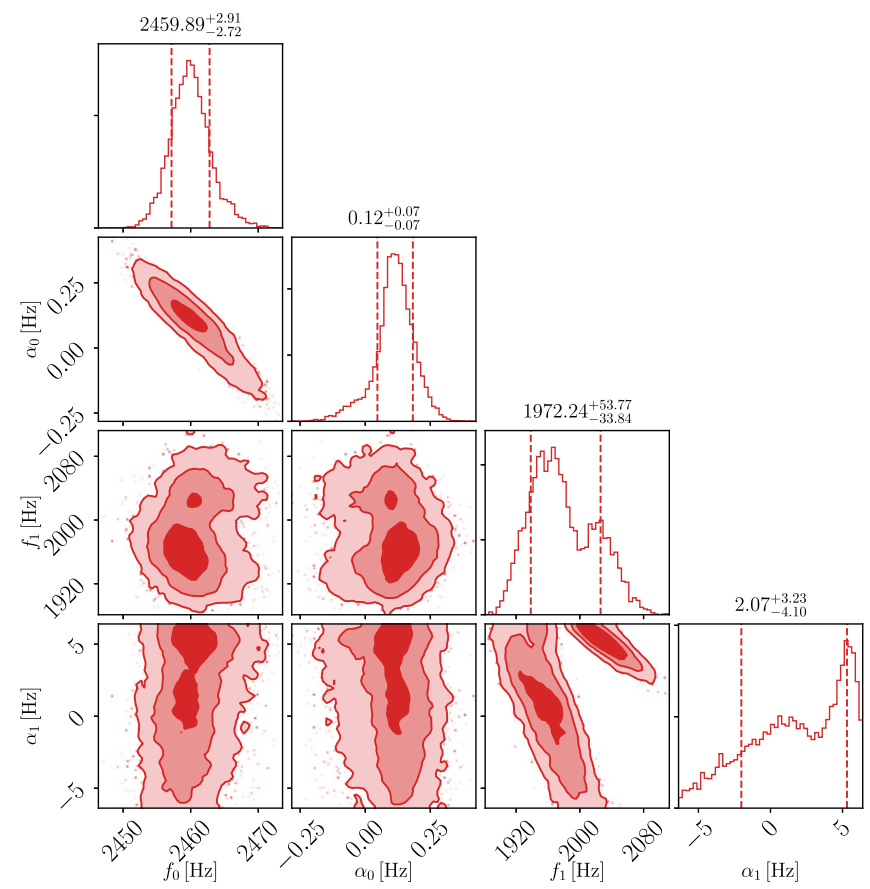

FIG. 12. As per Fig. 9 using the equation of state BHBlp with equal mass, $1.30 M_{\odot}$, neutron stars (waveform BHBlp-M1.300-

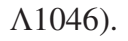

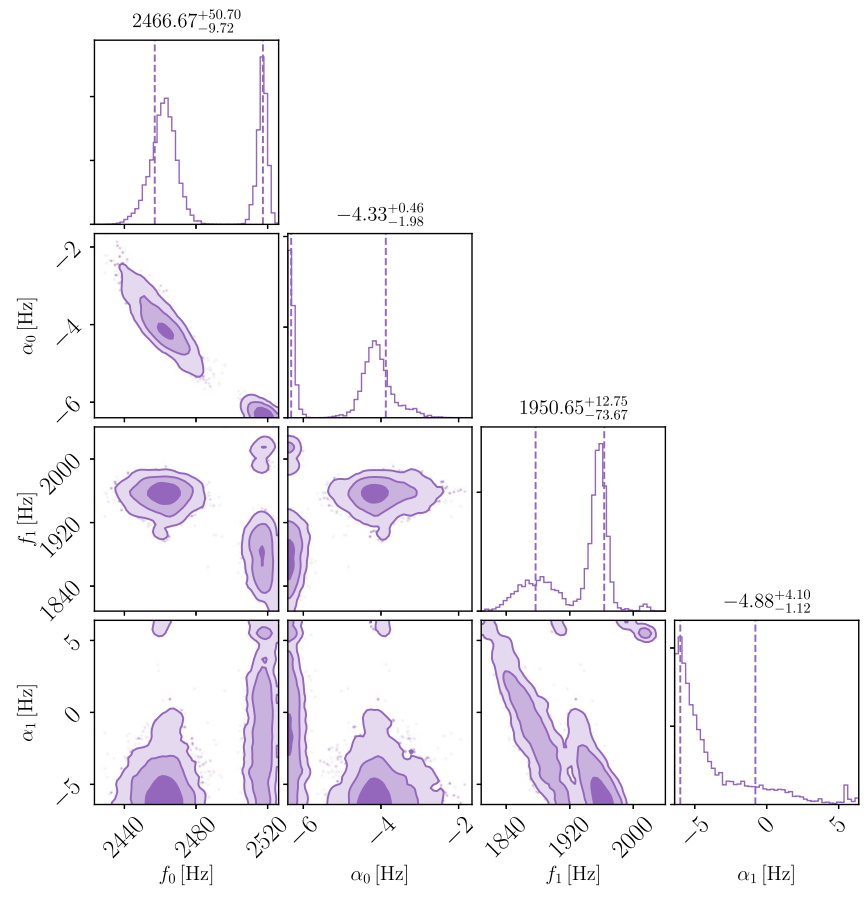

FIG. 13. As per Fig. 9 using the equation of state DD2 with equal mass, $1.25 M_{\odot}$, neutron stars (waveform DD2-M1.25011295). 


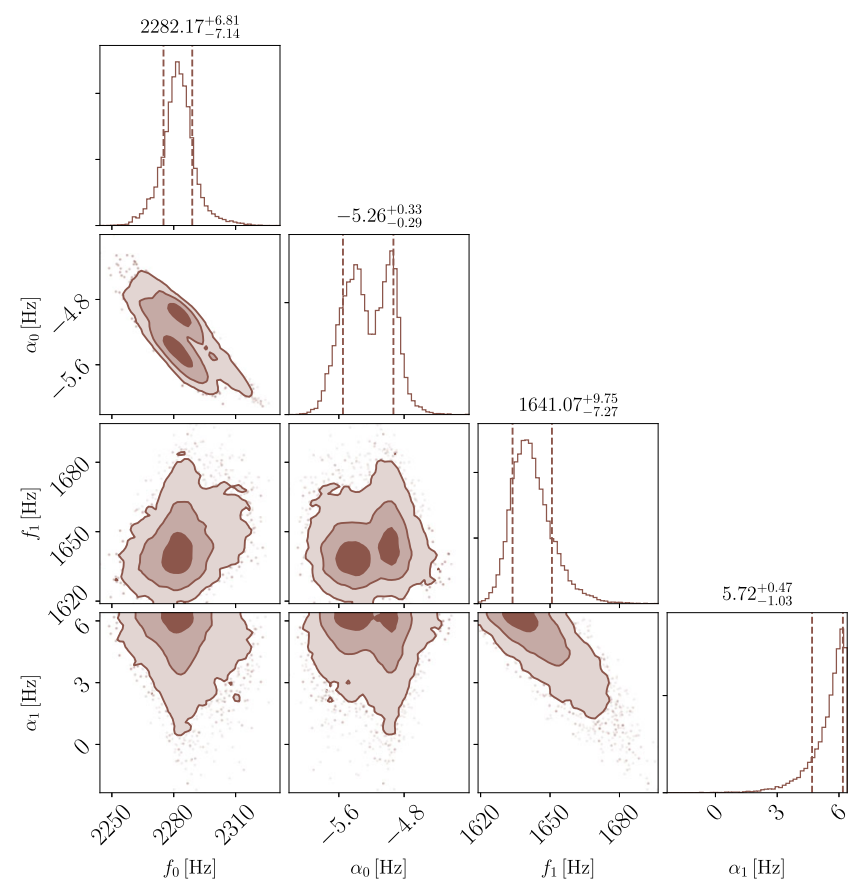

FIG. 14. As per Fig. 9 using the equation of state MS1b with equal mass, $1.375 M_{\odot}$, neutron stars (waveform MS1b-M1.375-

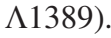

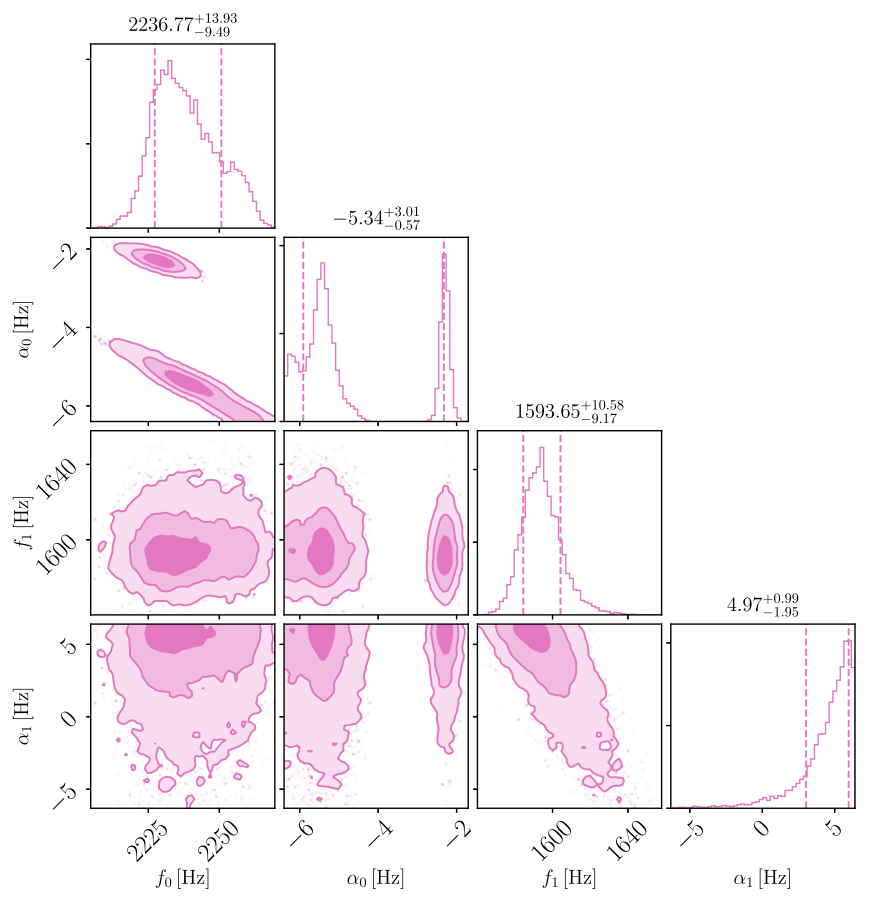

FIG. 15. As per Fig. 9 using the equation of state MS1b with equal mass, $1.35 M_{\odot}$, neutron stars (waveform MS1b-M1.35011532).

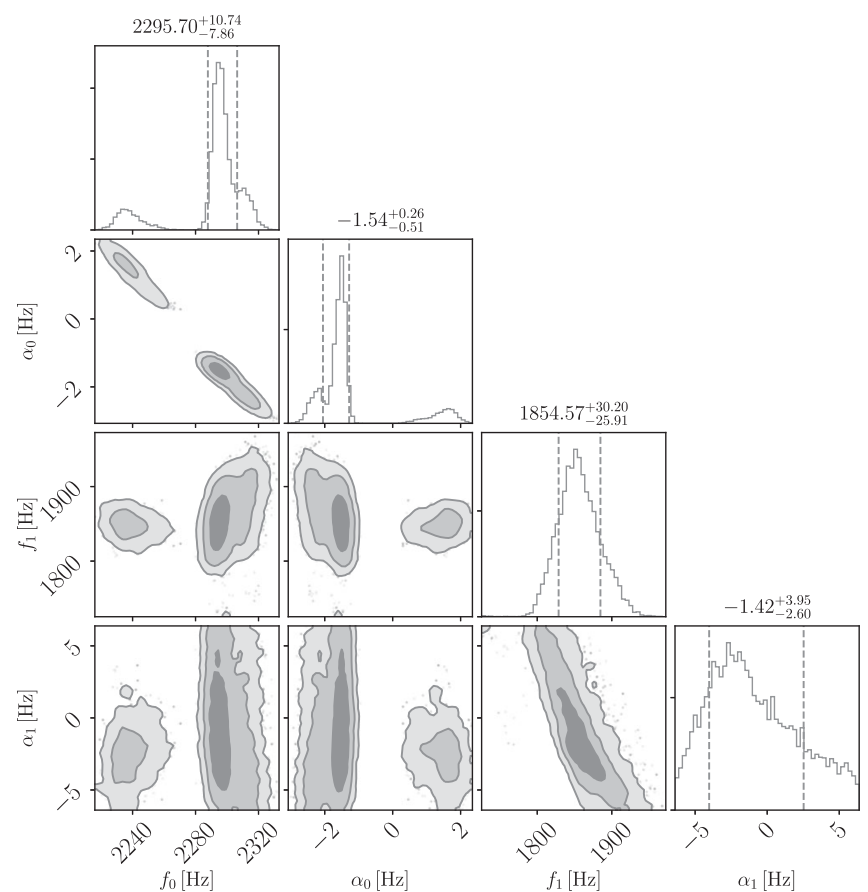

FIG. 16. As per Fig. 9 using the equation of state DD2 with equal mass, $1.20 M_{\odot}$, neutron stars (waveform DD2-M1.200-

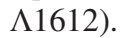

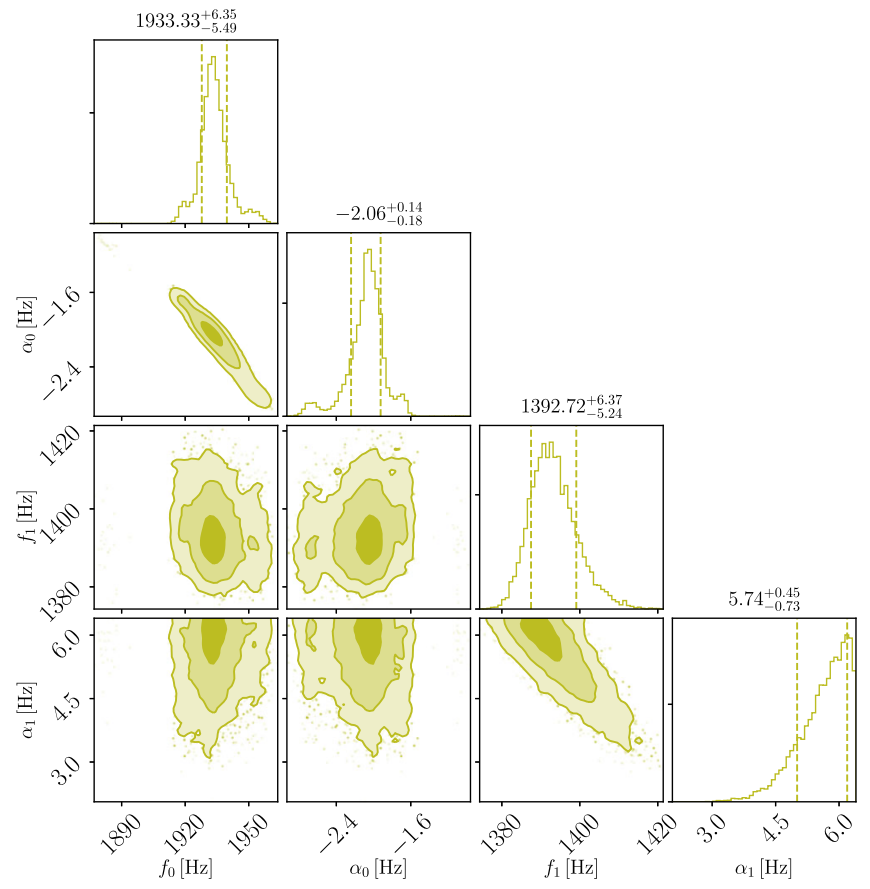

FIG. 17. As per Fig. 9 using the equation of state $2 \mathrm{H}$ with equal mass, $1.35 M_{\odot}$, neutron stars (waveform 2H-M1.350- 12326 ). 
[1] B. P. Abbott, R. Abbott, T. D. Abbott, F. Acernese, K. Ackley, C. Adams, T. Adams, P. Addesso, R. X. Adhikari, V. B. Adya et al., GW170817: Observation of Gravitational Waves from a Binary Neutron Star Inspiral, Phys. Rev. Lett. 119, 161101 (2017).

[2] B. P. Abbott, R. Abbott, T. D. Abbott, S. Abraham, F. Acernese, K. Ackley, C. Adams, R. X. Adhikari, V. B. Adya, C. Affeldt et al., GW190425: Observation of a compact binary coalescence with total mass $3.4 \mathrm{M}$, Astrophys. J. 892, L3 (2020).

[3] T. W. Baumgarte, S. L. Shapiro, and M. Shibata, On the maximum mass of differentially rotating neutron stars, Astrophys. J. 528, L29 (2000).

[4] M. Shibata, T. W. Baumgarte, and S. L. Shapiro, The barmode instability in differentially rotating neutron stars: Simulations in full general relativity, Astrophys. J. 542, 453 (2000).

[5] T. Nakamura, in Relativistic Cosmology, edited by M. Sasaki (Universal Academy Press, Tokyo, Japan, 1994), p. 155.

[6] K. C. B. New and J. E. Tohline, The relative stability against merger of close, compact binaries, Astrophys. J. 490, 311 (1997).

[7] F. A. Rasio and S. L. Shapiro, Coalescing binary neutron stars, Classical Quantum Gravity 16, R1 (1999).

[8] M. Shibata, K. Taniguchi, and K. Uryu, Merger of binary neutron stars with realistic equations of state in full general relativity, Phys. Rev. D 71, 084021 (2005).

[9] A. Bauswein and H.-T. Janka, Measuring Neutron-Star Properties Via Gravitational Waves from Neutron-Star Mergers, Phys. Rev. Lett. 108, 011101 (2012).

[10] A. Bauswein, H.-T. Janka, K. Hebeler, and A. Schwenk, Equation-of-state dependence of the gravitational-wave signal from the ring-down phase of neutron-star mergers, Phys. Rev. D 86, 063001 (2012).

[11] K. Hotokezaka, K. Kiuchi, K. Kyutoku, T. Muranushi, Y.-i. Sekiguchi, M. Shibata, and K. Taniguchi, Remnant massive neutron stars of binary neutron star mergers: Evolution process and gravitational waveform, Phys. Rev. D 88, 044026 (2013).

[12] S. Bernuzzi, A. Nagar, S. Balmelli, T. Dietrich, and M. Ujevic, Quasiuniversal Properties of Neutron Star Mergers, Phys. Rev. Lett. 112, 201101 (2014).

[13] K. Takami, L. Rezzolla, and L. Baiotti, Constraining the Equation of State of Neutron Stars from Binary Mergers, Phys. Rev. Lett. 113, 091104 (2014).

[14] S. Bernuzzi, T. Dietrich, and A. Nagar, Modeling the Complete Gravitational Wave Spectrum of Neutron Star Mergers, Phys. Rev. Lett. 115, 091101 (2015).

[15] A. Bauswein and N. Stergioulas, Unified picture of the postmerger dynamics and gravitational wave emission in neutron star mergers, Phys. Rev. D 91, 124056 (2015).

[16] K. Takami, L. Rezzolla, and L. Baiotti, Spectral properties of the postmerger gravitational-wave signal from binary neutron stars, Phys. Rev. D 91, 064001 (2015).

[17] L. Rezzolla and K. Takami, Gravitational-wave signal from binary neutron stars: A systematic analysis of the spectral properties, Phys. Rev. D 93, 124051 (2016).

[18] A. Bauswein, N.-U.F. Bastian, D. B. Blaschke, K. Chatziioannou, J. A. Clark, T. Fischer, and M. Oertel,
Identifying a First-Order Phase Transition in Neutron-Star Mergers Through Gravitational Waves, Phys. Rev. Lett. 122, 061102 (2019).

[19] M. Breschi, S. Bernuzzi, F. Zappa, M. Agathos, A. Perego, D. Radice, and A. Nagar, Kilohertz gravitational waves from binary neutron star remnants: Time-domain model and constraints on extreme matter, Phys. Rev. D 100, 104029 (2019).

[20] R. Oechslin and H.-T. Janka, Gravitational Waves from Relativistic Neutron-Star Mergers with Microphysical Equations of State, Phys. Rev. Lett. 99, 121102 (2007).

[21] M. Shibata, K. Taniguchi, and K. Uryu, Merger of binary neutron stars of unequal mass in full general relativity, Phys. Rev. D 68, 084020 (2003).

[22] L. Rezzolla, L. Baiotti, B. Giacomazzo, D. Link, and J. A. Font, Accurate evolutions of unequal-mass neutron-star binaries: Properties of the torus and short GRB engines, Classical Quantum Gravity 27, 114105 (2010).

[23] B. Giacomazzo, L. Rezzolla, and L. Baiotti, Accurate evolutions of inspiralling and magnetized neutron stars: Equal-mass binaries, Phys. Rev. D 83, 044014 (2011).

[24] K. Hotokezaka, K. Kyutoku, H. Okawa, M. Shibata, and K. Kiuchi, Binary neutron star mergers: Dependence on the nuclear equation of state, Phys. Rev. D 83, 124008 (2011).

[25] N. Stergioulas, A. Bauswein, K. Zagkouris, and H.-T. Janka, Gravitational waves and non-axisymmetric oscillation modes in mergers of compact object binaries, Mon. Not. R. Astron. Soc. 418, 427 (2011).

[26] A. Bauswein, N. Stergioulas, and H.-T. Janka, Exploring properties of high-density matter through remnants of neutron-star mergers, Eur. Phys. J. A 52, 56 (2016).

[27] S. Bose, K. Chakravarti, L. Rezzolla, B. S. Sathyaprakash, and K. Takami, Neutron-Star Radius from a Population of Binary Neutron Star Mergers, Phys. Rev. Lett. 120, 031102 (2018).

[28] P. J. Easter, P. D. Lasky, A. R. Casey, L. Rezzolla, and K. Takami, Computing fast and reliable gravitational waveforms of binary neutron star merger remnants, Phys. Rev. D 100, 043005 (2019).

[29] J. S. Read, L. Baiotti, J. D. E. Creighton, J. L. Friedman, B. Giacomazzo, K. Kyutoku, C. Markakis, L. Rezzolla, M. Shibata, and K. Taniguchi, Matter effects on binary neutron star waveforms, Phys. Rev. D 88, 044042 (2013).

[30] T. Dietrich, D. Radice, S. Bernuzzi, F. Zappa, A. Perego, B. Brueugmann, S. Vivekanandji Chaurasia, R. Dudi, W. Tichy, and M. Ujevic, CoRe database of binary neutron star merger waveforms and its application in waveform development, Classical Quantum Gravity 35, 24LT01 (2018).

[31] E. R. Most, L. J. Papenfort, V. Dexheimer, M. Hanauske, S. Schramm, H. Stöcker, and L. Rezzolla, Signatures of QuarkHadron Phase Transitions in General-Relativistic NeutronStar Mergers, Phys. Rev. Lett. 122, 061101 (2019).

[32] E. R. Most, L. R. Weih, L. Rezzolla, and J. SchaffnerBielich, New Constraints on Radii and Tidal Deformabilities of Neutron Stars from GW170817, Phys. Rev. Lett. 120, 261103 (2018).

[33] LIGO Scientific Collaboration, Advanced LIGO anticipated sensitivity curves, https://dcc.ligo.org/LIGO-T0900288/ public, ZERO_DET_high_P.txt (2010). 
[34] LIGO Scientific Collaboration, Prospects for observing and localizing gravitational-wave transients with Advanced LIGO, Advanced Virgo and KAGRA, https://dcc.ligo .org/LIGO-P1200087-v42/public, fig1_adv_sensitivity.txt (2016).

[35] G. Ashton et al., BILBY: A user-friendly Bayesian inference library for gravitational-wave astronomy, Astrophys. J. Suppl. 241, 27 (2019).

[36] J. S. Speagle, dynesty: A dynamic nested sampling package for estimating Bayesian posteriors and evidences, arXiv: 1904.02180.

[37] E. Annala, T. Gorda, A. Kurkela, and A. Vuorinen, Gravitational-Wave Constraints on the Neutron-Star-Matter Equation of State, Phys. Rev. Lett. 120, 172703 (2018).

[38] D. Radice, A. Perego, F. Zappa, and S. Bernuzzi, GW170817: Joint constraint on the neutron star equation of state from multimessenger observations, Astrophys. J. Lett. 852, L29 (2018).

[39] S. De, D. Finstad, J. M. Lattimer, D. A. Brown, E. Berger, and C.M. Biwer, Tidal Deformabilities and Radii of Neutron Stars from the Observation of GW170817, Phys. Rev. Lett. 121, 091102 (2018).

[40] B. P. Abbott, R. Abbott, T. D. Abbott, F. Acernese, K. Ackley, C. Adams, T. Adams, P. Addesso, R. X. Adhikari, V. B. Adya et al., Properties of the Binary Neutron Star Merger GW170817, Phys. Rev. X 9, 011001 (2019).

[41] T. A. Apostolatos, Search templates for gravitational waves from precessing, inspiraling binaries, Phys. Rev. D 52, 605 (1995).

[42] H. Jeffreys, Theory of Probability, 3rd ed. (Clarendon Press, Oxford, England, 1961).

[43] D. Martynov, H. Miao, H. Yang, F. H. Vivanco, E. Thrane, R. Smith, P. Lasky, W. E. East, R. Adhikari, A. Bauswein, A. Brooks, Y. Chen, T. Corbitt, A. Freise, H. Grote, Y. Levin, C. Zhao, and A. Vecchio, Exploring the sensitivity of gravitational wave detectors to neutron star physics, Phys. Rev. D 99, 102004 (2019).

[44] N. J. Cornish and T. B. Littenberg, Bayeswave: Bayesian inference for gravitational wave bursts and instrument glitches, Classical Quantum Gravity 32, 135012 (2015).

[45] T. B. Littenberg and N. J. Cornish, Bayesian inference for spectral estimation of gravitational wave detector noise, Phys. Rev. D 91, 084034 (2015).

[46] K. Chatziioannou, J. A. Clark, A. Bauswein, M. Millhouse, T. B. Littenberg, and N. Cornish, Inferring the postmerger gravitational wave emission from binary neutron star coalescences, Phys. Rev. D 96, 124035 (2017).

[47] A. Torres-Rivas, K. Chatziioannou, A. Bauswein, and J. A. Clark, Observing the postmerger signal of GW170817-like events with improved gravitational-wave detectors, Phys. Rev. D 99, 044014 (2019).

[48] T. Dietrich and T. Hinderer, Comprehensive comparison of numerical relativity and effective-one-body results to inform improvements in waveform models for binary neutron star systems, Phys. Rev. D 95, 124006 (2017).

[49] D. Radice, S. Bernuzzi, and C. D. Ott, One-armed spiral instability in neutron star mergers and its detectability in gravitational waves, Phys. Rev. D 94, 064011 (2016).

[50] C. Messenger, K. Takami, S. Gossan, L. Rezzolla, and B. S. Sathyaprakash, Source Redshifts from Gravitational-Wave Observations of Binary Neutron Star Mergers, Phys. Rev. X 4, 041004 (2014).

[51] K. W. Tsang, T. Dietrich, and C. Van Den Broeck, Modeling the postmerger gravitational wave signal and extracting binary properties from future binary neutron star detections, Phys. Rev. D 100, 044047 (2019).

[52] F. Zappa, S. Bernuzzi, D. Radice, A. Perego, and T. Dietrich, Gravitational-Wave Luminosity of Binary Neutron Stars Mergers, Phys. Rev. Lett. 120, 111101 (2018).

[53] T. Dietrich, S. Khan, R. Dudi, S. J. Kapadia, P. Kumar, A. Nagar, F. Ohme, F. Pannarale, A. Samajdar, S. Bernuzzi, G. Carullo, W. Del Pozzo, M. Haney, C. Markakis, M. Pürrer, G. Riemenschneider, Y. E. Setyawati, K. W. Tsang, and C. Van Den Broeck, Matter imprints in waveform models for neutron star binaries: Tidal and self-spin effects, Phys. Rev. D 99, 024029 (2019).

[54] https://www.gw-openscience.org.

[55] R. Pordes et al., The open science grid, J. Phys. Conf. Ser. 78, 012057 (2007).

[56] I. Sfiligoi, D. C. Bradley, B. Holzman, P. Mhashilkar, S. Padhi, and F. Wurthwrin, The pilot way to grid resources using glideinWMS, WRI World Congress 2, 428 (2009).

[57] D. Radice, General-relativistic large-eddy simulations of binary neutron star mergers, Astrophys. J. Lett. 838, L2 (2017).

[58] D. Radice, S. Bernuzzi, W. Del Pozzo, L. F. Roberts, and C. D. Ott, Probing extreme-density matter with gravitational-wave observations of binary neutron star merger remnants, Astrophys. J. Lett. 842, L10 (2017).

[59] F. Foucart, R. Haas, M. D. Duez, E. O'Connor, C. D. Ott, L. Roberts, L. E. Kidder, J. Lippuner, H. P. Pfeiffer, and M. A. Scheel, Low mass binary neutron star mergers: Gravitational waves and neutrino emission, Phys. Rev. D 93, 044019 (2016).

[60] T. Dietrich and K. Clough, Cooling binary neutron star remnants via nucleon-nucleon-axion bremsstrahlung, Phys. Rev. D 100, 083005 (2019). 